\title{
Mechanism of Membrane Recovery in Intra-Cytoplasmic Sperm Injection
}

\author{
Ricky Li ${ }^{1 *}$, Hao Chang ${ }^{2}$, Brian $\mathrm{Luo}^{3}$ \\ ${ }^{11}$ Department of Mechanical Engineering, University of Connecticut, CT, 06269, U.S.A \\ ${ }^{2}$ Watercare Services Limited, Auckland, 1050, New Zealand \\ ${ }^{3}$ Convergent Science, Madison, WI, 53719, U.S.A
}

\begin{abstract}
ICSI (Intra-cytoplasmic sperm injection) is a broadly utilized technique for artificial fertilization. This approach has been successfully performed in human oocytes as well as others such as mouse and bovine. The piercing through the zona layer and the membrane needs to be achieved with a minimal biological damage to facilitate a rapid healing. Since the injection methodology serves as a crucial factor to success rate of ICSI, a significant amount of research efforts has been devoted to the development of injections. In this paper, we conduct comparative study among the major milestones for injection techniques in ICSI. Technical details are provided for each milestone and each technique is evaluated from engineering perspective. Later, we present a mechanism for healing process of membrane after drilling, which could potentially provide guidance for improvement of injection method. More importantly, we perform coarsegrained molecular dynamics simulation to reveal the mechanism of membrane recovery in intracytoplasmic sperm injection.
\end{abstract}

Keywords: ICSI, Injection, Medical device, Piezo actuator, Ros-Drill, Piezo-ICSI.

\section{Introduction}

As a broadly utilized technique for artificial fertilization, ICSI (Intra-cytoplasmic sperm injection) has been successfully performed in human oocytes as well as others such as mouse and bovine [1-6]. Among the applications of ICSI, fertilization in mouse is of particular interest to biological and biomedical research. The significance of biological cell injection technology has attracted large amounts of research in an effort to automate laborious cell injection tasks. For the previous decade, many research efforts are dedicated to cell injection automation from a diverse array of aspects: cell holding devices, cell injection method, visionbased automation, cell injection process control, etc [7-8]. Conventional ICSI employs a spiked micropipette for facilitating penetration of the zona pellucida and piercing of the oolemma to enable penetration into the ooplasm and injection of whole sperm into the ovum. However, the irreversible damage to the ovum can be caused by the elasticity of the mouse oolemma, which makes it difficult to penetrate without rupture [9-10,63]. A minimal biological damage has to be achieved during the piercing through the zona layer and the membrane to facilitate a rapid healing [9]. A significant amount of research effort has been devoted to the development of ICSI from perspective of injection technique.

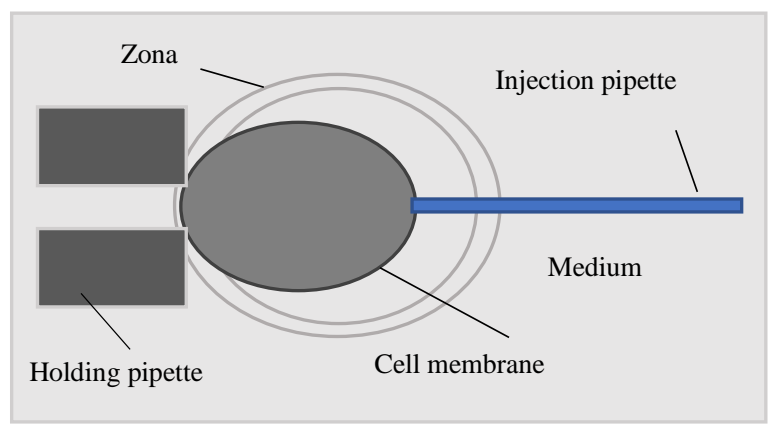

Fig. 1: Intra-cytoplasmic sperm injection setup. 
In conventional cellular injection for ICSI as shown in Fig. 1, an individual oocyte is immobilized by a holding pipette with a slight suction. The injection pipette containing the sperm head to be injected into the oocyte is forced into the cell. The survival rates and fertilization rates of oocytes for conventional ICSI vary from 80 to $90 \%$, and 45 to $70 \%$, respectively [11-16]. Size and sharpness of the needle used for injection are reported to be important for success rate and among the crucial factors is injection method of the needle into ooplasm [14].

Due to the low success rate and high labor intensity, piezo-ICSI was firstly employed to mice in 1995 [2530,64-66], which achieved high survival and fertilization rates in mice compared with those obtained by conventional ICSI. Despite the noticeably higher efficiency than conventional ICSI, in current practice, piezo-assisted ICSI requires the presence of a small amount of mercury to stabilize and suppress undesired lateral vibrations of the pipette tip under mechanical impact [17-20]. As of today, no other fluid material can replace mercury which can provide sufficiently high density necessary to mitigate these effects, preventing injection-induced damage to the ovum. Unfortunately, massive deployment of piezo-assisted ICSI in labs and institutions is prevented by the toxicity of mercury. Over the years, researchers have been taking great efforts to find alternative injection method to eliminate dependence on toxic substance in ICSI.

Later, a novel, mercury-free, rotationally oscillating drill (Ros-Drill) device was proposed as an alternative to piezo-assisted ICSI. Since it doesn't employ piezoelectric force actuator to drive a micropipette for performing ICSI in the mouse, it does not require mercury to suppress unwanted lateral motion of piezo. Preliminary results from Ros-Drill in mouse embryos was reported to be comparable to those of piezoassisted ICSI using mercury. Moreover, minimal demand on human expertise was claimed to be another benefit of this technology. These features result from the computer automated nature of the Ros-Drill technology as explained in the recent technology paper [21-23]. However, there is very few experimental or clinic data to support the statements and successful duplication by this method is necessary.

Most recent improvements in ICSI revolve around piezo-ICSI. Two main directions for improvement are micropipettes design to improve fertilization rate and injector design to avoid usage of mercury. For example, a piezoelectric driven non-toxic injector for automated cell manipulation was proposed [24], which claims to suppress detrimental lateral tip oscillation by centralizing the piezo oscillation power on the injector pipette However, so far only pipette movement is captured under high speed camera to show attenuation of lateral movement. Experimental and clinic data is not available now. Capability of piezoICSI may be compromised due to limiter applied in lateral dimension.

In this paper, at first, comparative study is presented for the major milestones for injection techniques in ICSI from extensive aspects. In essence, understanding of mechanism of membrane healing is crucial in ICSI from two-folds: a. new injection method to facilitate healing of membrane, $b$. facilitate healing after injection. At last, we present a mechanism for membrane healing process after injection.

\section{Major milestones}

In this section, technical details are provided for each milestone and each technique is evaluated from engineering perspective.

\subsection{Conventional ICSI}

The first method for ICSI is performed manually and is known as conventional ICSI [25-35]. With this technique, the tip of the injection pipette is approaching gently towards the cell membrane about halfway 
or even much further into the oocyte. Then it is pushed against membrane rapidly until it penetrates through the membrane. The spike-shaped pipette is most commonly used for conventional ICSI [26]. The high compliance of the mouse membrane makes the penetration very difficult without rupturing the ovum [14]. High failure rate for conventional ICSI can be attributed to the damage caused by the pressure of the injecting pipette on the cell membrane during the piercing process. Damage needs to heal properly and rapidly; otherwise abnormal growth occurs in the future stages of development $[14,32,44,67]$. Since manual operation can't perform piercing process quickly and accurately enough, deformation induced pressure inside membrane inevitably accumulates during piercing process. In light of limitation in manual operation, injection parameters such as injection depth (deformation level) and contact point become more critical and stringent, which requires extensive study in membrane modeling and intensive training in biologists and practitioners.

\subsection{Piezo-assisted ICSI}

The most popular procedure at the present is the piezo-assisted ICSI which has proved to increase the success rate beyond the conventional ICSI results [36-45]. In this technique, to generate minimal damage during the injection process, a piezo-activated axial force is applied to the injecting pipette to pierce smoothly the zona pellucida and then the cell membrane. Different from conventional ICSI, for piezoassisted ICSI penetration of the zona pellucida and oolemma can be facilitated with a flat-tipped (not spiked) micropipette [38]. In piezo-actuated micromanipulation, the piezo-electric effect is employed to transmit a small crystal lattice distortion to the tip of a pipette, moving it forward against membrane in a prescribed manner. Piezo-actuated micromanipulation has multiple applications in the study and engineering of gametes and embryos. For example, it enabled the first intracytoplasmic sperm injection (ICSI) to produce mice, the first nuclear transfer cloning of mice and pigs [41-42].

The typical controller parameters of piezo-assisted ICSI are set to $10 \mathrm{~V}$ amplitude, 60s duration, and $2 \mathrm{~Hz}$ frequency for mouse oocytes [36,46-48]. Introduction of piezo-actuation into ICSI for precise and consistent control is, in fact, a significant progress towards an effective automated deployment of microinjection operation. However, a small amount of mercury is usually placed near the tip of piezo-actuated pipette to suppress its transverse oscillations [17,18,42]. This high intensity substance can effective suppress vibration as the damping effect in lateral dimension.

The major supplier for Piezo-actuated micromanipulation are Eppendorf PiezoXpert and Burleigh $\odot$ Piezodrill. Usually, their devices enable tuning of parameters, such as speed and number of pulse and rampup/down slope for pulse [46,47]. These parameters need to be optimized after collections of experiments are conducted to achieve good results.

\subsection{Ros-Drill}

The main operational difference between piezo-drill and Ros-Drill is in the motion generation methods. Instead of the axial impact type action in the piezo-drill, the Ros-Drill rotationally oscillates the micropipette at a selected frequency and amplitude [21-22]. The intention for Ros-Drill design is that the ideal straight micropipette would rotationally oscillate about its axis without a lateral motion. However, the eccentricity in pipette due to manufacturing tolerance would generate some whirling motion during oscillation [21-22]. Therefore, the eccentricity level determines the unwanted lateral movement.

In alpha version of Ros-Drill, to avoid excessive lateral displacements, pipette is oscillated with very small angular amplitude ( $1^{\circ}$ peak-to-peak) and at frequencies that above natural frequencies of mode 1 and mode $2(90-100 \mathrm{~Hz})$ and much lower than the $3^{\text {rd }}$ mode [21]. $30^{\circ}$ bent pipettes are selected for during injection. 
This Ros-Drill microinjector prototype employs a PLC (Programmable Logic Controller) as the digital controller, which has a maximum sampling frequency of $1 \mathrm{kHz}$ [22]. This constraint limits the controllable trajectory to be at the maximum frequency of $500 \mathrm{~Hz}$ due to the Nyquist sampling rule. A DC servo motor is employed to actuate the pipette to track a sinusoidal position reference. The servo motor comes with an optical encoder with 512 lines and quadrature signature capability which could only provide a sensor resolution of $0.176^{\circ}[22]$.

Most recently reported ICSI tests use injection pipette oscillations up to $0.2^{\circ}$ amplitude and maximum frequency of $500 \mathrm{~Hz}$ [22]. Small angular oscillation is to minimize excessive lateral motion. High frequency is adopted to avoid cell membrane to follow the stimulus, facilitating a clean piercing process. One assumption is made in this new technique that the motion generated from piezo-actuator is faithfully transmitted to tip of pipette due to high rotational stiffness of the pipette holder and the pipette. Experiments were done to show that Ros-Drill operation generates smaller lateral oscillations at the tip, compared with the piezo-assisted ICSI cases [21-22]. Although biological results based on this prototype are very promising, the trajectory tracking performance of the alpha prototype is not satisfactory due to lowresolution of position sensor and low control sampling rate of the PLC.

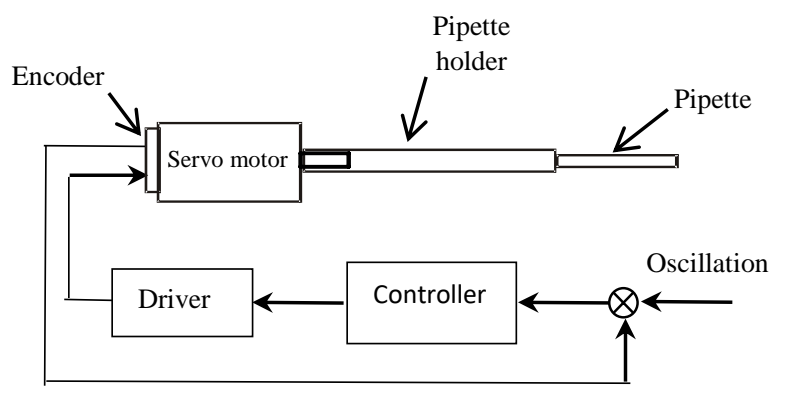

Fig. 2: Control system of Ros-Drill setup.

Beta version Ros-Drill as shown in Fig. 2 was designed and prototyped to be a high-precision, compact, and inexpensive setup. Essentially, servo motor is utilized to generate rotational oscillation and a closed loop control is implemented to ensure desired oscillation. Main improvements in hardware lie in microcontroller with sampling frequency of $10 \mathrm{kHz}$ and rotational motion sensor with resolution of $0.09^{\circ}$ [49-51]. Despite the improvement in hardware, resolution of encoder is still very low compared with target amplitude tracking $\left(0.2^{\circ}\right.$ amplitude). This is a typical mechatronic design with resource constraint, which stems from the position sensor in terms of size, resolution and costs demanded by the application [52-54]. Harmonic magnitude control is at the core of precision control and presents no particular difficulty when it uses appropriate sensors [55]. In order to track a $0.2^{\circ}$ amplitude oscillation with sensor with $0.09^{\circ}$ resolution, conventional control techniques fail to achieve desired tracking capability. Initially, a look-up table based gain scheduling technique was proposed to transform this low-resolution application to conventional proportional-integral-derivative (PID) method [49-50]. To counteract model uncertainty and disturbance in the control process, an adaptive control technique was designed to guarantee desired performance by adjusting control gains [56-57]. Moreover, an effective and systematic methodology which can achieve zero magnitude error tracking control for extremely low-resolution encoder was designed and implemented [58-61]. A stochastic stroke control problem was converted into a deterministic one by inferring actual motion from sensor signals [59]. The proposed control strategy consists of a two-stage adaptive control logic which adapts the control gains to achieve the actual peak-to-peak stroke. Comparisons of alpha and beta version of Ros-Drill are shown in Table 1. Controller and related peripherals are integrated into a control box. 
Table 1: Comparisons of alpha and beta version of Ros-Drill.

\begin{tabular}{|c|c|c|c|c|c|}
\hline & Controller & $\begin{array}{c}\text { Sampling } \\
\text { rate (kHz) }\end{array}$ & $\begin{array}{c}\text { Encoder } \\
\text { resolution (deg) }\end{array}$ & $\begin{array}{c}\text { Control } \\
\text { algorithm }\end{array}$ & $\begin{array}{c}\text { Trajectory tracking } \\
\text { capability (amplitude in deg) }\end{array}$ \\
\hline $\begin{array}{c}\text { Alpha } \\
\text { Ros-Drill }\end{array}$ & PLC & 1 & 0.18 & PID & 1 \\
\hline $\begin{array}{c}\text { Beta Ros- } \\
\text { Drill }\end{array}$ & $\begin{array}{c}\text { Microcont } \\
\text { roller }\end{array}$ & 10 & 0.09 & $\begin{array}{c}\text { Adaptive } \\
\text { control }\end{array}$ & 0.2 \\
\hline
\end{tabular}

Ros-Drill technique looks promising from drilling mechanism and implementation perspective. Somehow, very few institutions or clinics follow this protocol and very limited experimental data is available.

\subsection{Enhanced piezo-assisted ICSI}

\subsubsection{Micropipettes improvement}

Material and geometry of micropipettes also plays an important role in piezo-assisted ICSI. Researches and studies are conducted for optimization of geometry of micropipettes. It is shown that the Piezo-ICSI using micropipettes with a wall thickness of $0.625 \mu \mathrm{m}$ significantly improves the survival, fertilization, goodquality day-3 embryo, pregnancy, and live birth rates when compared to the conventional ICSI and PiezoICSI using micropipettes with a wall thickness of $0.925 \mu \mathrm{m}[62]$.

\subsubsection{Piezoelectric driven non-toxic injector}

In piezo-drill cell injection, mercury is placed at the tip of pipette to reduce generation of lateral tip oscillations of injection pipette. Some alternative is proposed to suppress this excessive lateral motion in piezo-driven cell injection. A new design for piezo-actuated injector eliminates unwanted oscillations by applying physical constraints on the pipette [24]. It doesn't rely on mercury to attenuate destructive lateral tip oscillations of the injector pipette. This mercury-free injector can sublime the piezoelectric driven injection technique to completely non-toxic level [24].

Essentially, the new injector employs a rigid attachment to limit lateral motion from piezo actuator. The efficacy of this limiter is demonstrated by the suppression of lateral movement under high speed camera by $50 \%$ [24]. However, no experimental or clinical data is available for this newly proposed method. From engineering perspective, additional constraint on actuator in one dimension usually compromises actuation capabilities of other dimensions due to mechanical coupling. Piercing force from axial oscillation of piezoelectric device is inevitably attenuated as a consequence. More simulation/modeling, e.g. finite element analysis and experiments need to be performed to demonstrate efficacy of this technique.

\section{Methodology comparison}

This section provides comparison of three major injection methods, i.e. conventional, piezo-assisted and Ros-Drill, as shown in Table 2. Conventional injection is manual and labor-intensive compared with piezoassisted and Ros-Drill. From actuation perspective, piezo provides axial vibration whereas Ros-Drill rotational oscillation. In spite of availability of commercialized medical device for conventional ICSI, satisfactory success rate still calls for intensive training in biologists and practitioners. Advanced control algorithm and mechatronic design enable Ros-Drill to achieve desired motion at low cost, which puts RosDrill at a favorable position in contrast to Piezo-assisted device. The statistics for publications pertinent to injection methods of ICSI are based on data from 1995 to 2017. 
Nowadays, based on number of related publications and deployment, piezo-assisted method dominates in experimental and clinic activities in ICSI. In terms of survival and fertilization rate, Ros-Drill and Piezoassisted method are comparable. However, since much more experimental data is available for Piezoassisted method than Ros-Drill, the survival and fertilization rate for Piezo-assisted method is more statistically significant. Again Ros-Drill technique look promising from drilling mechanism and proposed implementation perspective. Somehow, very few institution or clinics follow this protocol and very limited experimental data is available.

Table 2: Comparisons between three major injection techniques for ICSI.

\begin{tabular}{|c|c|c|c|c|c|c|c|}
\hline & $\begin{array}{c}\text { Motion } \\
\text { type }\end{array}$ & $\begin{array}{c}\text { Survival } \\
\text { rate }(\%)\end{array}$ & $\begin{array}{c}\text { Fertilization } \\
\text { rate }(\%)\end{array}$ & $\begin{array}{c}\text { Cost } \\
(\$ \mathrm{k})\end{array}$ & Drawback & $\begin{array}{c}\text { \# Publication } \\
(1995-2017)\end{array}$ & $\begin{array}{c}\# \\
\text { Usages }\end{array}$ \\
\hline Conventional & Manual & 80 & 45 to 70 & N/A & $\begin{array}{c}\text { labor } \\
\text { intensive }\end{array}$ & $>300$ & 500 \\
\hline Piezo & Axial & 90 & 75 & $>5$ & mercury & 85 & 300 \\
\hline Ros-Drill & $\begin{array}{c}\text { Rotational } \\
\text { oscillation }\end{array}$ & 70 & 80 & 0.6 & $\begin{array}{c}\text { insufficient } \\
\text { data }\end{array}$ & 18 & N/A \\
\hline
\end{tabular}

\section{Mechanism of membrane recovery after drilling}

In essence, understanding of mechanism of membrane healing is crucial in ICSI from two-folds: a. new drilling method to facilitate membrane healing, b. method to facilitate healing after drilling.

The cell membrane, also called plasma membrane, is very thin with thickness of a few molecules. The major role of cell membrane is to isolate the intracellular contents. In addition, the cell membrane also serves to maintain the shape of the cells. Another function of the membrane is to control intercellular transport via endocytosis and exocytosis. The cargo lipids and proteins are moved from the cell membrane as substances are internalized through endocytosis whereas vesicles containing lipids and proteins fuse with the cell membrane to cargo in important proteins and enzymes. The cell membrane primarily consists of a mix of membrane proteins and lipids. The occupation of lipids can range from 20 to 80 percent of the membrane, relying on the cell type and their roles. Lipids help to maintain membranes flexibility, membrane proteins regulate the cell's chemical environment and promote the transfer of molecules via the membrane. Phospholipids are a major component of cell membranes. Phospholipids form a bilayer where their hydrophilic head areas are oriented to the extracellular fluid, whereas their hydrophobic tail stay away from the cytosol and extracellular fluid. The lipid bilayer is semi-permeable. It allows limited molecules to diffuse through the membrane. Cholesterol is another major lipid component of cell membranes. Cholesterol molecules are largely distributed between membrane phospholipids to prevent cell membranes from stiffening through keeping phospholipids from being packed together. Glycolipids present on cell membrane surfaces with function of helping the cell to identify other cells of the body. The cell membrane contains two distinct types of associated proteins as well. Peripheral membrane proteins are exterior to the membrane. They are connected to membrane by binding with other proteins. Integral membrane proteins are inserted into the membrane, exposing on both sides of the membrane. Cell membrane proteins have various functions. Structural proteins play an essential role in the cell support and shape. Cell membrane receptor proteins is vital for cells communication with their external environment through the use of hormones, neurotransmitters, and other signaling molecules. Transport proteins of globular proteins, transport molecules across cell membranes through diffusion. Glycoproteins are embedded in the cell membrane with a carbohydrate chain attached to them, which contributes the cell communications and molecule transport across the membrane. 
The membrane does not contribute to the cell's mechanical strength, which is attributed to the cytoskeleton or cell wall. In addition to the lipid bilayer and integral proteins, RBC membrane possesses a 2D cytoskeleton tethered to the lipid bilayer. The RBC membrane cytoskeleton has a 2D six-fold structure, and is made of the spectrin tetramers connected at the actin junctional complexes, forming a $2 \mathrm{D}$ six-fold structure. The cytoskeleton is connected to the lipid bilayer via the "immobile" band-3 proteins at the spectrin-ankyrin binding sites and the glycophorin protein at the actin junctional complexes.

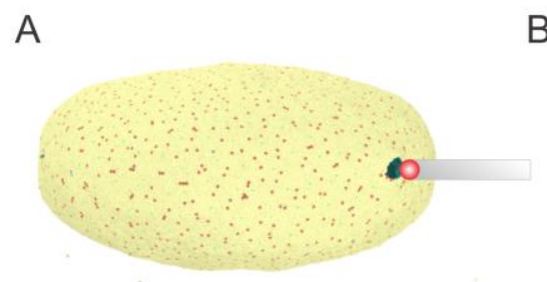

B

C
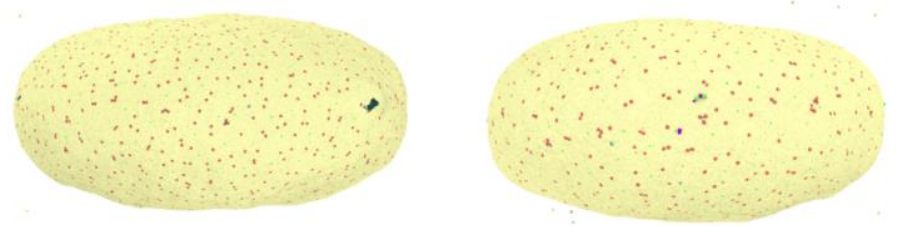

Fig.3 Healing of cell membrane after withdrawing the micropipette.

Membrane can heal after drilling. Once the micropipette is withdrawn, the lipid molecules rearrange themselves to reduce exposure of their hydrophobic regions to water. However, the rearrangement of the lipids at the pore boundary results in configurations that are not as energetically favorable as the bilayer itself, such that there is an energy penalty for the formation of holes. This energy penalty can be described by a line tension or edge tension, which is an energy per unit length along the boundary of the hole. The effective edge tension is temperature-dependent and vanishes at sufficiently high temperature, where the membrane is unstable against hole formation even in the absence of mechanical stress. Therefore, it drives the lipid molecules to the hole to reduce the line tension until the membrane is healed. We perform coarse-grained molecular dynamic simulation to illustrate the healing process, as shown in Fig.3. In the applied cell model, $\mathrm{CG}$ particles are introduced to represent lipid bilayer and transmembrane proteins (see Fig. 3). The CG particles carry both translational and rotational degrees of freedom $\left(\mathbf{x}_{i}, \mathbf{n}_{i}\right)$, where $\mathbf{x}_{\mathrm{i}}$ and $\mathbf{n}_{\mathrm{i}}$ are the position and the orientation (direction vector) of particle $i$, respectively. The rotational degrees of freedom obey the normality condition $\left|\mathbf{n}_{\mathrm{i}}\right|=1$. Thus, each CG particle effectively carries 5 degrees of freedom. $\mathbf{x}_{\mathrm{ij}}=\mathbf{x}_{\mathrm{j}}-\mathbf{x}_{\mathrm{i}}$ is defined as the distance vector between particles $i$ and $j$. Correspondingly, $r_{\mathrm{ij}} \equiv\left|\mathbf{x}_{\mathrm{ij}}\right|$ is the distance, and $\hat{\mathbf{x}}_{\mathrm{ij}}=\mathbf{x}_{\mathrm{ij}} / r_{\mathrm{ij}}$ is a unit vector. The CG particles, forming the lipid membrane and membrane proteins, interact with one another via a pair-wise additive potential

$$
u_{\text {mem }}\left(\mathrm{n}_{\mathrm{i}}, \mathrm{n}_{\mathrm{j}}, \mathrm{x}_{\mathrm{ij}}\right)=u_{\mathrm{R}}\left(r_{\mathrm{ij}}\right)+A\left(\alpha, a\left(\mathrm{n}_{\mathrm{i}}, \mathrm{n}_{\mathrm{j}}, \mathrm{x}_{\mathrm{ij}}\right)\right) u_{\mathrm{A}}\left(r_{\mathrm{ij}}\right),
$$




$$
\begin{cases}u_{R}\left(r_{i j}\right)=k \varepsilon\left(\left(R_{c u t, m e m}-r_{i j}\right) /\left(R_{c u t, m e m}-r_{e q}\right)\right)^{8}-k \varepsilon & \text { for } \quad r_{i j}<R_{c u t, \text { mem }} \\ u_{A}\left(r_{i j}\right)=-2 k \varepsilon\left(\left(R_{c u t, m e m}-r_{i j}\right) /\left(R_{c u t, m e m}-r_{e q}\right)\right)^{4}-k \varepsilon & \text { for } \quad r_{i j}<R_{c u t, \text { mem }} \\ u_{R}\left(r_{i j}\right)=u_{A}\left(r_{i j}\right)=0, & \text { for } \quad r_{i j} \geq R_{c u t, \text { mem }}\end{cases}
$$

The healing process is highly dependent on the mechanical state of the membrane. When the membrane is under swelling (hydrated), it becomes thinner and its hydrophobic core is increasingly exposed to water so it ruptures, releasing tension through increasing the hole such that the cell becomes ruptured. On the other hand, when the membrane is under compression (dehydrated cell), the lipid molecules tend to migrate to the hole to release the compression and thus accelerate the healing the membrane. Therefore, we hypothesis that the dehydrated examined cells will facilitate healing after drilling. Our above discussions are based on the analysis of the mechanics of the lipid bilayer and cytoskeleton. We plan to validate our hypothesis by performing numerical simulation through using particle-based cell membrane and fiber models [68-77] with appropriate description of hydrodynamic interactions [78-79]. We also hope that these discussions can potentially stimulate and steer new experiments in this area.

\section{Conclusion}

A significant amount of research effort has been devoted to injection method for ICSI to achieve minimal biological damage to facilitate a rapid healing of membrane. Major milestones for injection techniques in ICSI is summarized. Their comparisons are also presented from engineering perspective. Piezo-assisted method is dominant in ICSI activities, around which further improvement in ICSI is built. Ros-Drill technique looks promising from drilling mechanism and proposed implementation perspective. However, very few institution or clinics follow this protocol and very limited experimental data is available. At last, we present a mechanism for healing process of membrane after drilling, which could potentially provide guidance for improvement of injection method.

\section{References}

[1] D. Dozortsev, T. Wakayama, A. Ermilov and R. Yanagimachi, "Intracytoplasmic sperm injection in the rat", Zygote, vol.6 (2), May 1998.

[2] A. Fonttis, R. Napolitano and M.A. Tomas, "Successful ICSI in a case of severe asthenozoospermia due to $93 \%$ non-specific axonemal alterations and $90 \%$ abnormal or absent mitochondrial sheaths", Reprod. Biomed. Online, vol.5 (3) pp.270-272, 2002.

[3] G. Palermo, H. Joris, P. Devroey and A.C. Van Steirteghem, "Pregnancies after intracytoplasmic injection of single spermatozoon into an oocyte", Lancet, vol.340, July 1992.

[4] M. Plachot, J. Belaisch-Allart, J.M. Mayenga, A. Chouraqui, L. Tesquier and A.M. Serkine, "Outcome of conventional IVF and ICSI on sibling oocytes in mild male factor infertility", Human Reproduction, vol.17 (2) pp.362-369, 2002. 
[5] R. Suttner, V. Zakhartchenko, P. Stojkovic, S. Muller, R. Alberio, I. Medjugorac, G. Brem, E. Wolf and M. Stojkovic, "Intracytoplasmic sperm injection in bovine: effects of oocyte activation, sperm pretreatment and injection technique", Theriogenology, vol.54 (6) pp. 935-948, 2000.

[6] Z. Zhang and N. Olgac, "An Adaptive Control Method for Ros-Drill Cellular Microinjector with Lowresolution Encoder", Journal of Medical Engineering, vol. 2013, issue 1, pp. 1-11, 2013.

[7] W.H. Wang, et al., "A fully automated robotic system for microinjection of zebrafish embryos", PLoS ONE, vol. 2, no. 9, 2007.

[8] H. B. Huang, et al., "Robotics cell injection system with vision and force control: Towards automatic batch biomanipulation", IEEE Trans. on Robotics, vol. 25, no. 3, pp. 727-737, 2009.

[9] Kimura Y and Yanagimachi R, "Intracytoplasmic Sperm Injection in the Mouse", Biol Reprod, vol. 52, pp. 709-720, 1995.

[10]Deng M, Kishikawa H, Yanagimachi R, Kopf GS, Schultz RM, Williams CJ, "Chromatin-mediated cortical granule redistribution is responsible for the formation of the cortical granule-free domain in mouse eggs", Dev Biol, vol. 257, issue 1, pp. 166-76, 2003.

[11] Payne, D., Flaherty, P., Jeffrey, R. et al, "Successful treatment of severe male factor infertility in 100 consecutive cycles using intracytoplasmic sperm injection", Hum. Reprod., vol. 9, pp. 2051-2057, 1994.

[12] Sherins, R.J., Thorsell, L.P., Dorfmann, A. et al, "Intracytoplasmic sperm injection facilitates fertilization even in the most severe forms of male infertility: pregnancy outcome correlates with maternal age and number of eggs available", Hum. Reprod., vol. 64, pp. 369-375, 1995.

[13] Tucker, M.J., Wright, G., Morton, P.C. et al, "Practical evolution and application of direct intracytoplasmic sperm injection for male factor and idiopathic fertilization failure infertilities", Fertil. Steril., vol. 63, pp. 820-827, 1995.

[14] Vanderzwalmen, P., Bertin, G., Lejeune, B. et al, "Two essential steps for a successful intracytoplasmic sperm injection: injection of immobilized spermatozoa after rupture of the oolemma", Hum. Reprod., vol. 11, pp. 540-547, 1996.

[15] Gao, Q. and Olgac, N. "Stability Analysis for LTI Systems with Multiple Time Delays Using the Bounds of its Imaginary Spectra", Systems \& Control Letters, 102, pp.112-118, 2017.

[16] Gao, Q. and Olgac, N. "Determination of the Bounds of Imaginary Spectra of LTI Systems with Multiple Time Delays", Automatica, 72, pp. 235-241, 2016.

[17]K. Ediz, N. Olgac, "Micro-dynamics of the piezo-driven pipettes in ICSI", Biomedical Engineering, IEEE Transactions on, Volume:51, Issue:7, July 2004 Pages:1262 - 1268.

[18] K. Ediz, N. Olgac, "Effect of Mercury Column on the Microdynamics of the Piezo-Driven Pipettes", ASME Journal of Biomechanical Engineering, Vol. 127, pp. 531-535, June 2005.

[19] Gao, Q., Kammer, A.S., Zalluhoglu, U. and Olgac, N. "Critical Effects of the Polarity Change in Delayed States Within an LTI Dynamics With Multiple Delays", IEEE Transactions on Automatic Control, 60 (11), pp. 3018-3022, 2015.

[20]Gao, Q., Kammer, A.S., Zalluhoglu, U. and Olgac, N. "Combination of Sign Inverting and Delay Scheduling Control Concepts for Multiple-delay Dynamics”, Systems \& Control Letters, 77, pp. 55-62, 2015.

[21]Ergenc, A.F. and Olgac, N., "New Technology for Cellular Piercing: Rotationally Oscillating Microinjector, Description and Validation Tests", J. Biomedical Microdevices, vol. 9, no. 6, pp: 885-891, 2007.

[22]Ergenc, A.F., Li, M., Toner, M., Biggers, J., Kent, J.C. and Olgac, N., "Rotationally Oscillating Drill (Ros-Drill()) for Mouse ICSI without using Mercury”, Mol. Reprod. Dev. 1-8, 2008.

[23]Z. Zhang and N. Olgac. "Adaptive Gain Scheduling for Rotationally Oscillating Drill (Ros-Drill), with Low-Resolution Feedback", International Journal of Mechatronics and Manufacturing Systems, vol. 6, issue 5/6, pp. 397-421, 2013.

[24]B. Huang, S. Hao and Y. Chen, "Piezoelectric driven non-toxic injector for automated cell manipulation", Studies in health technology and informatics, vol. 163, pp. 231-5, 2011. 
[25] Van Steirteghem, A.C., Nagy, P., Joris, H. et al, “The development of intracytoplasmic sperm injection”, Hum. Reprod., vol. 8, pp. 59-72, 1996.

[26] P. Collas and F.L. Barnes, "Nuclear transplantation by microinjection of inner cell mass and granulosacell nuclei”, Molecular Reproduction and Development, vol.38 (3) pp. 264-267, July 1994.

[27] Gao, Q. and Olgac, N. "Optimal Sign Inverting Control for Time-delayed Systems, A Concept Study with Experiments", International Journal of Control, 88 (1), pp.113-122, 2015.

[28]Gao, Q., Zalluhoglu, U. and Olgac, N. "Investigation of Local Stability Transitions in the Spectral Delay Space and Delay Space", ASME Journal of Dynamic Systems, Measurement and Control, 136 (5), pp. 051011, 2014.

[29] M. Bonduelle, I. Liebaers, V. Deketelaere, M.P. Derde, M. Camus, P. Devroey and A.V. Steirteghem, "Neonatal data on a cohort of 2889 infants born after ICSI (1991-1999) and of 2995 infants born after IVF (1983-1999)", Human Reproduction, vol.17 (3) pp. 671-694, 2002.

[30] X. Zhang, S. Wang, J. B. Hoagg, and T. M. Seigler, "The roles of feedback and feedforward as humans learn to control unknown dynamic systems", IEEE Transactions on Cybernetics, 2017.

[31] Y. Kawase, T. Iwata, Y. Toyoda, T. Wakayama, R. Yanagimachi and H. Suzuki, "Comparison of intracytoplasmic sperm injection for inbred and hybrid mice", Molecular Reproduction and Development, vol.60 pp.74-78, 2001.

[32]P. Devroey A. Van Steirteghem, "A review of ten years experience of ICSI", Human Reproduction Update, vol. 10, issue 1, pp. 19-28, 2004.

[33]X. Zhang and J. B. Hoagg, "Subsystem identification of multivariable feedback and feedforward systems," Automatica, vol. 72, pp. 131-137, 2016.

[34] Gao, Q., Cepeda-Gomez, R. and Olgac, N. "A Test Platform for Cognitive Delays: Target Tracking Problem with Multiple Time-delayed Feedback Control", International Journal of Dynamics and Control, 2 (1), pp. 77-85, 2014.

[35] Parrington, J., Swann, K., Shevchenko, V.I. et al, "Calcium oscillations in mammalian eggs triggered by a soluble sperm protein", Nature, vol. 379, pp. 364-368, 1996.

[36] Yoshida N and Perry ACF, "Piezo-actuated Mouse Intracytoplasmic Sperm Injection (ICSI)", Nature Protocols, vol. 2, no. 2, pp. 296-304, 2007.

[37] T. Huang, Y. Kimura and R. Yanagimachi, "The use of piezo micromanipulation for intracytoplasmic sperm injection of human oocytes", Journal of Assisted Reproduction and Genetics, vol.13 (4) pp. 320328, 1996.

[38] H. Katayose, K. Yanagida, T. Shinoki, T. Kawahara, T. Horiuchi and A. Sato, "Efficient injection of bull spermatozoa into oocytes using a piezo-driven pipette", Theriogenology, vol.52 pp.1215-1224, 1999.

[39] Q. Gao, Z. Zhang and C. Yang, "Sign Inverting Control and its Important Properties for Multiple TimeDelayed Systems", in Proceedings of 2017 ASME Dynamic Systems and Control Conference, Tysons Corner, Virginia, Oct. 11-13, 2017.

[40] X. Zhang and J. B. Hoagg, "Frequency-domain subsystem identification with application to modeling control strategies used by humans," Systems \& Control Letters, vol. 87, pp. 36-46, 2016.

[41] Baltaci V, Ayvaz OU, Unsal E, Aktas Y, Baltaci A, Turhan F, Ozcan S, Sonmezer M, "The effectiveness of intracytoplasmic sperm injection combined with piezoelectric stimulation in infertile couples with total fertilization failure”, Fertil Steril, vol. 94, pp. 900-904, 2010.

[42] K. Yanagida H. Katayose H. Yazawa Y. Kimura K. Konnai A. Sato, "The usefulness of a piezomicromanipulator in intracytoplasmic sperm injection in humans", Human Reproduction, vol. 14, issue 2, pp. 448-453, 1999.

[43] X. Zhang, T. M. Seigler, and J. B. Hoagg, "Modeling the control strategies that humans use to control nonminimum-phase systems," Proceedings of the American Control Conference, pp. 471-476, Chicago, IL, July 2015. 
[44] Montag M, Koster M, van der Ven K, Bohlen U, van der Ven H, "The benefit of artificial oocyte activation is dependent on the fertilization rate in a previous treatment cycle", Reprod Biomed Online, vol. 24, pp. 521-526, 2012.

[45]Jie Luo, Chengyu Cao, Naira Hovakimyan, "L1 Adaptive Controller for a Class of Systems with Unknown Nonlinearities", Proceedings of 2010 American Control Conference, pp. 1659-1664, 2010.

[46] Stein P and Schneider I. Piezo-actuated Mouse ICSI. Eppendorf Userguide 032. www.eppendorf.com.

[47] Nagy A, Gerstenstein M, Vintersten K, Behringer, B. Manipulating the Mouse Embryo: A Laboratory Manual (Third Edition). Cold Spring Harbor Laboratory Press; 2003.

[48] J. Luo and C. Cao, "Consensus in multi-agent systems with nonlinear uncertainties under a fixed undirected graph," International Journal of Control, Automation and Systems, vol. 12, no. 2, pp. 231-240, 2014.

[49]Z. Zhang and N. Olgac, "An Adaptive Control Method with Low-Resolution Encoder", Proceedings of 2013 ASME Dynamic Systems and Control Conference, Stanford Univ., CA, Oct. 21-23, 2013.

[50]Z. Zhang, J. Diaz and N. Olgac, "Adaptive Hybrid Control for Rotationally Oscillating Drill (RosDrill()), Using a Low-Resolution Sensor", Proceedings of 2011 ASME Dynamic Systems and Control Conference, Arlington, Virginia, Oct. 31-Nov. 2, 2011.

[51]Luo, J., Cao, C., and Yang, Q., "L1 Adaptive controller for a class of non-affine multi-input multioutput nonlinear systems", International Journal of Control, 86, 348-359, 2013.

[52] Shan Tang, Zhenyu Zhang, Chuanwen Ji, Duc T. Phan, "Data storage device demodulating servo burst by computing slope of intermediate integration points," U.S. patent US9286927, March 15, 2016.

[53] Alexander Babinski, Zhenyu Zhang, Eugene Chun-Tsing Wu, Alan M. Fossen, Chuanwen Ji, Duc T. Phan, Donald Brunnett, "Electronic system with media scan mechanism and method of operation thereof," U.S. patent US9349401, May 24, 2016.

[54] Yanan Huang, Zhenyu Zhang, Chuanwen Ji, Duc T. Phan, "Disk drive measuring stroke difference between heads by detecting a difference between ramp contact," U.S. patent US9355666, May 31, 2016.

[55]Z. Zhang and Q. Gao, "Characterization of Extreme Low-Resolution Digital Encoder for Control System with Sinusoidal Reference Signal”, 19th International Conference on Networking, Sensing and Control (ICNSC), Tokyo, Japan, May 28-29, 2017.

[56]Z. Zhang, J. Diaz and N. Olgac, "Adaptive gain scheduling for rotationally oscillating drill, with lowresolution feedback", International Journal of Mechatronics and Manufacturing Systems, vol. 6, issue 5/6, pp. 397-421, 2013.

[57] J. Luo, C. Cao, "L adaptive output feedback controller for a class of nonlinear systems", 50th IEE Conference on Decision and Control, Orlando, FL, pp. 5425-5430, 2011.

[58]Z. Zhang and N. Olgac, "Zero Magnitude-Error Tracking Control for Servo System with Extremely Low-resolution Digital Encoder", International Journal of Mechatronics and Manufacturing Systems, in press, 2017.

[59]Z. Zhang, N. Olgac and Q. Gao, "An effective algorithm to achieve accurate sinusoidal amplitude control with a low-resolution encoder", in Proceedings of IEEE International Conference on Advanced Intelligent Mechatronics, Munich, Germany, July 3-7, 2017.

[60] Gao, Q. and Olgac, N. "Optimal Sign Inverting Control for Multiple Time-delayed Systems". 2016 American Control Conference. Boston, MA, USA, July 6-8, 2016.

[61] X. Zhang, S. Wang, T. M. Seigler, and J. B. Hoagg, "Frequency-domain observations on how humans learn to control an unknown dynamic system," Proceedings of the American Control Conference, pp. 1143-1148, Chicago, IL, July 2015.

[62] Kenichiro Hiraoka and Seiji Kitamura, "Clinical efficiency of Piezo-ICSI using micropipettes with a wall thickness of 0.625 $\mu \mathrm{m}$ ", J Assist Reprod Genet, vol. 32(12), pp. 1827-33, 2015.

[63] Kang-Decker N, Mantchev GT, Juneja SC, McNiven MA, van Deursen JM, "Lack of acrosome formation in Hrb-deficient mice", Science, vol. 294, pp. 1531-1533, 2001.

[64] Lewis SE, Aitken RJ, "DNA damage to spermatozoa has impacts on fertilization and Pregnancy", Cell Tissue Res, vol. 322, pp. 33-41, 2005. 
[65]Hoshi, K., Yanagida, K., Yazawa, H. et al., "Intracytoplasmic sperm injection using immobilized or motile human spermatozoon", Fertil. Steril., vol. 63, pp. 1241-1245, 1995.

[66] HsiehYY, Chang CC,Tsai HD, "Zona-free oocyte fertilized with intracytoplasmic sperm injection and underwent further division: case report and literature review", Chang Gung Med J, vol. 24, pp. 599602, 2001.

[67] Moaz MN, Khattab S, Foutouh IA, Mohsen EA, "Chemical activation of oocytes in different types of sperm abnormalities in cases of low or failed fertilization after ICSI: a prospective pilot study", Reprod Biomed Online, vol. 13, pp. 791-794, 2006.

[68] Li, H. and Lykotrafitis, G. A coarse-grain molecular dynamics model for sickle hemoglobin fibers. Journal of the mechanical behavior of biomedical materials, 4(2), 162-73, 2011

[69] Li, H.,Ha, V. and Lykotrafitis, G. Modeling sickle hemoglobin fibers as one chain of coarse-grained particles. Journal of biomechanics, 45(11), 1947-51, 2012

[70] Li, H. and Lykotrafitis, G. Two-component coarse-grained molecular-dynamics model for the human erythrocyte membrane. Biophysical journal, 102(1), 75-84, 2012

[71] Li, H. and Lykotrafitis, G. Erythrocyte membrane model with explicit description of the lipid bilayer and the spectrin network. Biophysical journal, 107(3), 642-53, 2014

[72] Li, H. and Lykotrafitis, G. Vesiculation of healthy and defective red blood cells. Physical Review E, 92(1), 012715, 2015

[73] Li, H.,Zhang, Y.,Ha, V. and Lykotrafitis, G. Modeling of band-3 protein diffusion in the normal and defective red blood cell membrane. Soft matter, 12(15), 3643-53, 2016

[74] Li, X.,Li, H.,Chang, H.-Y.,Lykotrafitis, G. and Karniadakis, G. E. Computational biomechanics of human red blood cells in hematological disorders. Journal of Biomechanical Engineering, 139(2), 021008, 2017

[75]Lu, L.,Li, H.,Bian, X.,Li, X. and Karniadakis, G. E. Mesoscopic Adaptive Resolution Scheme (MARS) toward understanding of interactions between sickle cell fibers. Biophysical Journal, 113(1), 48-59, 2017

[76] Tang, Y.-H.,Lu, L.,Li, H.,Evangelinos, C.,Grinberg, L.,Sachdeva, V., et al. OpenRBC: A Fast Simulator of Red Blood Cells at Protein Resolution. Biophysical Journal, 112(10), 2030-7, 2017

[77]Chang, H.-Y.,Li, X.,Li, H. and Karniadakis, G. E. MD/DPD multiscale framework for predicting morphology and stresses of red blood cells in health and disease. PLoS Comput Biol, 12(10), e1005173, 2016

[78] Yang, J. A Smoothed Dissipative Particle Dynamics Methodology For Wall-Bounded Domains. Ph.D Dissertation: Worcester Polytechnic Institute (2013).

[79] Gatsonis, N. A.,Potami, R. and Yang, J. A smooth dissipative particle dynamics method for domains with arbitrary-geometry solid boundaries. Journal of Computational Physics, 256, 441-64 (2014) 